\title{
Factores institucionales y satisfacción laboral del profesional de enfermería del Hospital María Auxiliadora, Lima
}

\author{
Institutional factors and job satisfaction of the nursing professional of the Hospital María \\ Auxiliadora, Lima.
}

Nancy Huamán Salazar*

\begin{abstract}
RESUMEN
Objetivo: Determinar la relación entre los factores institucionales y la satisfacción laboral del profesional de enfermería del Hospital María Auxiliadora. Material y Método: Investigación con enfoque cuantitativo, de diseño no experimental, descriptivo correlacional y de corte transversal. La muestra estuvo conformada por 130 profesionales de enfermería que laboran en los diferentes servicios del Hospital María Auxiliadora. La técnica de recolección de datos fue la encuesta y el instrumento el cuestionario elaborado por la investigadora. Resultados: Muestran que el 93.1\% de la muestra pertenece al sexo femenino y el 6.9\% al sexo masculino; el 43\% tiene más de 40 años de edad, el 22.3\% entre 35 y 40 años y el 34.7\% tiene menos de 35 años; el $44.6 \%$ es casado y el 42.3\% soltero; en cuanto al tiempo de servicio en la institución, el 34.6\% labora menos de 5 años, el 32.3\% de 5 a 14 años y el $33.1 \%$ más de 15 años. Existe relación directa y significativa entre los factores institucionales y la satisfacción laboral ( $\mathrm{Rho}=0.272, \mathrm{p}=0.002)$. También se encontró que los factores institucionales de salario (Rho=0.378, $\mathrm{p}=0.000$ ), condiciones laborales $(\mathrm{Rho}=0.227, \mathrm{p}=0.009)$ y políticas administrativas (Rho=0.234, $\mathrm{p}=0.007)$ tienen una relación directa y significativa con la satisfacción laboral. Sin embargo, los factores de supervisión $(\mathrm{Rho}=0.059, \mathrm{p}=0.502)$ y relaciones interpersonales ( $\mathrm{Rho}=-0.012, \mathrm{p}=0.888)$ no presentan evidencia estadística de relación con la satisfacción laboral. El análisis de relación se hizo a través del coeficiente de correlación de Spearman. Conclusiones: La relación directa y significativa del salario, condiciones laborales y políticas administrativas representan la satisfacción; no así, la supervisión y relaciones interpersonales, que, previenen la insatisfacción.
\end{abstract}

Palabras clave: Satisfacción laboral, motivación, factores institucionales.

\begin{abstract}
Objetive: This study determines the relationship between institutional factors and job satisfaction of the nursing professional at Maria Auxiliadora Hospital. Material and Methods: It is a quantitative research approach, nonexperimental, correlational and cross-sectional descriptive. The sample consisted of 130 nurses working in the various departments of Maria Auxiliadora Hospital. The data collection technique was survey and the instrument a questionnaire prepared by the researcher. Results: The results show that $93.1 \%$ of the sample are female and $6.9 \%$ males. Age percentages are $43 \%$ over 40 years old, $22.3 \%$ between 35 and 40 years and $34.7 \%$ are under 35. Regarding marital status, $44.6 \%$ are married and $42.3 \%$ single. In respect to time of service in the institution, $34.6 \%$ have worked less than 5 years, 32.3\% 5 to 14 years and 33.1\% over 15 years. There is a direct and significant relationship between institutional factors and job satisfaction $($ Rho $=0.272, p=0.002)$. It was also found that institutional factors wage (Rho $=0.378, \mathrm{p}=0.000)$, working conditions $(\mathrm{Rho}=0.227, \mathrm{p}=0.009)$ and administrative policies $(\mathrm{Rho}=0.234, \mathrm{p}=0.007)$ have a direct and significant relationship with work satisfaction. However, supervising factors (Rho $=0.059, \mathrm{p}=0.502$ ) and interpersonal relationships (Rho $=-0,012, \mathrm{p}=0.888$ ) have no statistical evidence regarding job satisfaction. Ratio analysis was through the Spearman's rank correlation coefficient. Conclusions: Direct and significant relationship of wages, working conditions and administrative policies, represent satisfaction; not, supervision and interpersonal relationships, which prevent dissatisfaction.
\end{abstract}

Keywords: Job satisfaction, motivation, institutional factors. 


\section{INTRODUCCIÓN}

Las organizaciones de salud, para lograr una gestión que trascienda, deben dar importancia a la satisfacción del personal que labora, pues si este está satisfecho, eleva su rendimiento y productividad. La satisfacción laboral es una percepción subjetiva e individual producto de una valoración afectiva de las personas frente a su trabajo (Spector, 1997).

En el ámbito laboral existen factores que inciden en la satisfacción del personal, dentro de los cuales están los factores externos como el salario, las condiciones laborales, la supervisión, relaciones interpersonales y políticas administrativas. (Balderas 2009). Estudios de los factores que afectan la satisfacción laboral en Estados Unidos (Chen-Chung 2007), España, México, Chile, Venezuela y Brasil (Cifuentes 2012), son similares y concordantes a factores higiénicos.

En el Perú, se promueve acciones para fortalecer las capacidades del personal y mejorar la calidad en la atención del usuario. No obstante, el personal se preocupa por ofrecer servicios de excelencia y por lograr mejorar sus competencias, pero se siente desmotivado y amenazado por factores muchas veces externos que limitan su actuar laboral. Por ello, es necesario que los equipos de gestión reconozcan qué factores inciden en la satisfacción del personal a fin de modificarlos o controlarlos.

El objetivo del estudio fue determinar la relación entre los factores institucionales y la satisfacción laboral del profesional de enfermería, para obtener resultados fidedignos que permitan orientar a los directivos sobre estrategias de motivación y satisfacción del personal, a fin de lograr mayor eficiencia y eficacia en los servicios y por ende elevar calidad del cuidado al usuario. Las enfermeras más satisfechas tienden a estar más comprometidas con las organizaciones en donde se desempeñan (Umayma, Yaktin; Azoury, Doumit, Myrna 2003).

\section{MATERIALES Y MÉTODOS}

Es una investigación con enfoque cuantitativo, tipo básica, de campo. El diseño de la investigación fue no experimental, de corte transversal y descriptiva correlacional.

El estudio se realizó en el Hospital María Auxiliadora, institución de III nivel. La población estuvo conformada por todos los enfermeros que laboran en los servicios de enfermería, la muestra obtenida por muestreo probabilístico aleatorio simple, el tamaño estimado de 128.82 , con un nivel de confianza del 95\% y una precisión del 3\%, se decidió trabajar con 130 profesionales de enfermería. El muestreo aleatorio obtenido de una lista de enfermeros, codificados e ingresados al programa SPSS, donde se eligieron a 130 por aleatoriedad del software, se tuvo en cuenta criterios de inclusión (experiencia laboral mayor de un año y participar voluntariamente) y exclusión (no deseen participar, estar con licencia o enfermedad).

La técnica utilizada fue la encuesta, el instrumento un cuestionario que comprende los datos generales de edad, género, servicio donde labora, tiempo de servicio y estado civil; y los datos específicos que se organizaron en dos secciones: la primera sección con 22 ítems sobre los factores institucionales, graduados según la escala de Likert, totalmente de acuerdo (TA $)=5$, acuerdo $(\mathrm{A})=4$, indeciso $(\mathrm{I})=3$, desacuerdo (D)=2 y totalmente en desacuerdo (TD)=1; la segunda sección 11 ítems sobre la satisfacción laboral, se valoran de acuerdo a la escala: nada satisfecho=1, poco satisfecho $=2$, bastante satisfecho $=3$ y muy satisfecho=4. La validez se realizó a través de 07 jueces, la validez del contenido según tabla binomial de concordancia de jueces expertos, obteniendo un valor $\mathrm{p}=0,03$. La confiabilidad se realizó según muestra piloto, previo consentimiento informado. Se aplicó la prueba alfa de Cronbach obteniendo un puntaje de 0.892 para los factores institucionales y 0.855 para la satisfacción laboral se demostró que el instrumento es confiable.

El procesamiento y análisis de información se realizó mediante el paquete estadístico SPSS Versión 22, para la comprobación de los supuestos la prueba paramétrica de correlación de Pearson, al no cumplirse con el supuesto de normalidad se procedió a evaluar la correlación a través de la prueba no paramétrica de correlación de Spearman. Los resultados de correlación se presentan en una tabla cruzada considerando el valor $\mathrm{n}$ de pares, el valor del coeficiente de correlación de Spearman (Rho) y su respectivo p valor. 


\section{RESULTADOS}

Tabla 1

Características generales de los profesionales de enfermería del Hospital María Auxiliadora, 2015

\begin{tabular}{|c|c|c|}
\hline Variables y categorías & $\mathrm{n}$ & $\%$ \\
\hline \multicolumn{3}{|l|}{ Género } \\
\hline Femenino & 121 & 93.1 \\
\hline Masculino & 9 & 6.9 \\
\hline \multicolumn{3}{|l|}{ Edad } \\
\hline Menor de 25 años & 1 & 0.8 \\
\hline De 25 a 29 años & 21 & 16.2 \\
\hline De 30 a 34 años & 23 & 17.7 \\
\hline De 35 a 40 años & 29 & 22.3 \\
\hline Mayor de 40 años & 56 & 43.0 \\
\hline \multicolumn{3}{|l|}{ Estado civil } \\
\hline Soltero & 55 & 42.3 \\
\hline Casado & 58 & 44.6 \\
\hline Viudo & 4 & 3.1 \\
\hline Conviviente & 8 & 6.2 \\
\hline Divorciado & 5 & 3.8 \\
\hline \multicolumn{3}{|l|}{ Servicio donde labora } \\
\hline Emergencia & 20 & 15.4 \\
\hline Hospitalización & 75 & 57.7 \\
\hline Consultorios Externos & 6 & 4.6 \\
\hline Preventivo Promocional & 5 & 3.8 \\
\hline Centro Quirúrgico & 22 & 16.9 \\
\hline Otros & 2 & 1.5 \\
\hline \multicolumn{3}{|l|}{ Tiempo de servicio } \\
\hline Menor de 5 años & 45 & 34.6 \\
\hline De 5 a 9 años & 22 & 16.9 \\
\hline De 10 a 14 años & 20 & 15.4 \\
\hline De 15 a 19 años & 10 & 7.7 \\
\hline De 20 a 25 años & 14 & 10.8 \\
\hline Mayor de 25 años & 19 & 14.6 \\
\hline
\end{tabular}

La Tabla 1 presenta las características generales de los 130 profesionales de enfermería que participaron en el estudio, de los cuales el 93.1\% de enfermeros son mujeres y el $6.9 \%$ varones; el $43 \%$ tiene más de 40 años de edad, el $22.3 \%$ entre 35 y 40 años y el 34.7\% tiene menos de 35 años; con respecto al estado civil, los que tuvieron mayor representatividad fueron los casados con $44.6 \%$, seguido de los solteros con $42.3 \%$ y con $6.2 \%$, $3.8 \%$ y $3.1 \%$ el estado civil de conviviente, divorciado y viudo respectivamente. El 57.7\% labora en los Servicios de Hospitalización, el 16.9\% en Centro Quirúrgico el 15.4\% en Emergencia, el 4.6\% en Consultorios Externos, el 3.8\% en Preventivo Promocional y el 1.5\% en otros servicios. En cuanto al tiempo de servicio en la institución, el 34.6\% labora menos de 5 años, el 32.3\% de 5 a 14 años y el 33.1\% más de 15 años.

Tabla 2

Relación entre los factores institucionales y la satisfacción laboral del profesional de enfermería del Hospital María Auxiliadora, 2015

\begin{tabular}{llll}
\hline Variables & \multicolumn{3}{l}{ Satisfacción Laboral } \\
\cline { 2 - 4 } & $\mathrm{n}$ & $\mathrm{Rho}$ & $\mathrm{p}$ \\
\hline Factores institucionales & 130 & 0.272 & 0.002 \\
Factor salario & 130 & 0.378 & 0.000 \\
Factor condiciones laborales & 130 & 0.227 & 0.009 \\
Factor supervisión & 130 & 0.059 & 0.502 \\
Factor políticas administrativas & 130 & 0.234 & 0.007 \\
Factor relaciones interpersonales & 130 & -0.012 & 0.888 \\
\hline
\end{tabular}

La Tabla 2 presenta los resultados del análisis de correlación entre las variables de estudio, se evidencia una relación directa y significativa entre los factores institucionales y la satisfacción laboral $(\mathrm{Rho}=0.272$, $\mathrm{p}=0.002$ ), se muestra que existe una relación directa y significativa de los factores: salario $(\mathrm{Rho}=0.378$, $\mathrm{p}=0.000)$, condiciones laborales $(\mathrm{Rho}=0.227, \mathrm{p}=0.009)$ y políticas administrativas $(\mathrm{Rho}=0.234, \mathrm{p}=0.007)$ con la satisfacción laboral; sin embargo, no se evidencia relación directa y significativa de los factores: supervisión (Rho $=0.059, p=0.502)$ y relaciones interpersonales (Rho=-0.012, $\mathrm{p}=0.888)$ con la satisfacción laboral.

\section{DISCUSIÓN}

Existen factores institucionales que pueden condicionar una situación, ya sea de satisfacción o insatisfacción, por ello, es importante identificarlos a fin de poder modificarlos o controlarlos, para esto es necesario conocer los hábitos de pensamientos y de acción compartidos entre los miembros de la institución (Veblen, 1899 citado por Nelson, 2001).

En el presente estudio se encontró que existe relación directa y significativa entre los factores institucionales: salario, condiciones laborales y políticas administrativas con la satisfacción laboral del profesional de enfermería, sin embargo, no se evidencia relación estadística significativa entre los factores institucionales de supervisión y relaciones interpersonales, con la satisfacción laboral. 
La relación directa y significativa observada entre el factor salario y la satisfacción laboral, de los profesionales de enfermería, significa que ellos lo consideran como un factor muy importante para sentirse bien en su área laboral, por tanto, los directivos de una organización deberían conocer estas apreciaciones del grupo de enfermeros a fin de motivarlos, considerando que el salario es definido como la cantidad de signos monetarios que recibe la enfermera y depende de las características del trabajo y del trabajador (Perazi, 2007).

El salario representa la principal forma de compensación organizacional, es una contraprestación por el trabajo de una persona en la organización (Ivancevich et al., 2006). Según Chiavenato (2002) para las personas el trabajo es un medio de alcanzar un objetivo intermedio, por ello es importante señalar que el salario es la fuente de renta que da poder adquisitivo. El pago que el empleado recibe de la organización es el más importante elemento de su poder adquisitivo; el volumen de dinero que una persona gana también sirve como indicador de poder y prestigio, lo que influye en sus sentimientos de autoestima. La remuneración afecta a las personas desde el punto de vista económico, sociológico y psicológico (Chiavenato, 2002 citado por Iona, Iturbe y Osorio, 2013).

Según la teoría Microeconómica Convencional, los niveles más altos de salario permiten niveles más elevados de consumo y están asociados con niveles altos de satisfacción laboral. Por otro lado, se consideran las variaciones compensadoras asociadas a diferentes características no monetarias del empleo a partir de estimaciones del nivel de bienestar subjetivo de los individuos (Gómez, 2012). Estas medidas compensadoras conocidas también como aspectos intangibles incluyen la confianza y el respeto, la posibilidad de aprender nuevas habilidades, el sentirse involucrado en la toma de decisiones y percibir el agradecimiento por un trabajo bien hecho, que, en muchos casos, producen mayor satisfacción (Schultz, 1995, citado por Ramírez et al., 2008). En los profesionales de enfermería, es importante este factor porque les permite gozar de un mejor nivel de vida personal y familiar compensando el arduo trabajo que realiza y los riesgos a los que están expuestos. Por ello, los gerentes deben establecer estrategias de vincular las recompensas con el desempeño y los trabajadores deben percibir la relación entre ambas, si los individuos perciben que la relación es débil, los resultados serán un desempeño deficiente, una disminución en la satisfacción laboral y un aumento en la rotación del personal y el ausentismo (Robbins 2013).

En el estudio, los resultados también muestran que el factor condiciones laborales tiene relación directa y significativa con la satisfacción laboral de los profesionales de enfermería, coincidiendo con lo vertido por Herrera (2008) que las condiciones de trabajo guardan estrecha relación con la satisfacción e insatisfacción laboral y es uno de los factores relacionados con la motivación, indispensable en la calidad del servicio ofertado por la enfermera. Por otro lado, Ponce (2006), citado por Cisneros (2011), encontró que las deficiencias en las condiciones de trabajo específicamente en (áreas físicas y falta de material y equipo) afecta notablemente la satisfacción del personal de enfermería, coincidiendo con este estudio que existe una relación directa y significativa.

Estos factores involucran la seguridad del lugar de trabajo, los pasadizos, los pisos, las conexiones de los equipos, la comodidad y el confort, además lo constituye la iluminación, ruido, temperatura, recursos físicos y materiales (OIT citado por Cisneros 2011). Por ello Molina (2009) refiere que se debe mejorar las condiciones de trabajo, con el afán de mejorar la calidad de los cuidados como meta final.

En el área de enfermería, las condiciones laborales constituyen un riesgo para su salud física y mental, por cuanto el ejercicio de cuidar implica una sobrecarga laboral y emocional, debido a los múltiples problemas que derivan de la atención y de los sistemas de salud, así como a sus propias exigencias personales, profesionales y familiares; en este orden de ideas, los profesionales de enfermería ven amenazada su satisfacción laboral y profesional cuando las condiciones son adversas (Leguizamón y Gómez, 2002). Por tanto, es importante unir esfuerzos para mejorar las condiciones laborales a fin de proteger su salud, garantizar la satisfacción del personal y, por ende, un mayor rendimiento y producción laboral.

En el presente estudio también se demuestra que las políticas institucionales tienen relación directa y significativa con la satisfacción laboral; de esta manera, se afirma que la gestión es congruente con el desarrollo profesional. Es importante señalar que el trabajador tiene la necesidad de sentirse valorado, atendido, escuchado y recompensado por los directivos. De esta manera, se configura el entramado de fuerzas que convierten a una empresa en competitiva y exitosa (Bellido 2000) para la cual las políticas deben incluir estos aspectos positivos para los trabajadores. Si bien las políticas son los medios para cumplir con metas y objetivos (Marriner 1993), estas deben orientar la toma de decisiones (Balderas 2009) que involucren la capacitación y perfeccionamiento, remuneraciones, prevención de riesgos, evaluación del desempeño y desvinculación (Méndez 2009). 
El análisis de relación, del presente estudio, reportó valor no significativo entre el factor supervisión y la satisfacción laboral de los profesionales de enfermería. Estos resultados probablemente se deban a la influencia de una variable interviniente que no fue controlada, pues se aplicó el cuestionario inmediatamente después de un período de cambios en la estructura organizativa con potencial efecto en las percepciones del personal. Por tanto, se sugiere estudiar este factor en un momento de mayor estabilidad organizativa. En ese sentido el profesional de enfermería en su precepción no solo ve a la supervisora como un miembro directivo, sino también percibe su actitud frente al personal, donde el nivel de comunicación que existe debe ser coherente y permanente. Sin embargo, un estudio de Briseño (2008) citado por Martínez (2009) en Argentina, midió la satisfacción laboral en el personal de enfermería y encontró que la “relación con los jefes y superiores”, una mayoría se mostraron pocos satisfechos.

Estos factores influyen en el desempeño; no obstante, se situarían al margen del contenido del trabajo (factores extrínsecos), puesto que no son motivadores por sí mismos, sino que tienden a evitar la insatisfacción, significa que el trabajar con supervisión cercana no es muy motivador y es aún más desmotivador trabajar sin supervisión, lo que significa que para los empleados es necesario que los responsables del área les proporcionen las directrices para sus actividades.

Si bien la supervisión refleja una función influyente en el recurso humanos, esta no está siendo valorada por el personal de enfermería, pues, se dice que una supervisión es eficaz, cuando el resultado es la satisfacción personal, la productividad y la calidad del trabajo. Sin duda que la función de la supervisión constituye un enlace como mediador e integrador de los diferentes cursos de acción en el proceso de atención al paciente, puesto que el bienestar del paciente implica la agrupación en tiempo y forma de tareas asistenciales y de criterios de racionalización de la gestión (Vinagre 2013).

En este estudio se puede apreciar la necesidad de reajustar o replantearse la función que cumple una enfermera supervisora, que permita motivar para lograr satisfacción y de mejorar la apreciación que la enfermera tiene respecto a su desempeño. En cuanto al factor de relaciones interpersonales, estas influyen en la satisfacción laboral, como lo demuestra Edwards (2009) en un estudio donde encontró que la confianza en las relaciones interpersonales tiene una influencia positiva y significativa en la satisfacción laboral. Un componente fundamental, de las relaciones interpersonales en el trabajo, es la confianza in- terpersonal (Tan y Lim, 2009 citado por Omar 2011) que es considerada como un facilitador de la relación y las actitudes efectivas en el trabajo (Ferres, Connell y Travaglione, 2004).

Es importante mencionar que cuando un personal está satisfecho mejora la productividad del trabajo $\mathrm{y}$, por ende, las relaciones interpersonales. Cuando una enfermera asume una postura de forma flexible, moviliza y asume distintas funciones y roles, utiliza al máximo sus habilidades, conocimientos y recursos para ayudar al enfermo (Arredondo 2009). Uno de los principales factores que influyen en la capacidad de la enfermera para manejar la interacción y solucionar sus problemas podría radicar en la experiencia, pues garantiza que esta tenga confianza en sí misma, tenga habilidad en la relación y habilidad en la comunicación (Ortega 2007).

Por otro lado, Luthans (2008) menciona que las relaciones interpersonales con supervisores y compañeros se han clasificado como factores que pueden influir en experimentar insatisfacción laboral y tendrían escaso impacto en generar satisfacción laboral en los trabajadores. En el presente estudio, no se encontró relación significativa entre el factor relaciones interpersonales y la satisfacción laboral, probablemente por la misma variable interviniente que se mencionó en el factor anterior. Además, puede explicarse con la afirmación de Luthans, que este factor está mayormente ligado a la insatisfacción laboral.

Los datos obtenidos indican que los directivos de la institución deben poner atención y buscar estrategias para mejorar las relaciones interpersonales a través de la confianza y la comunicación, ya que esto va a determinar eficiencia y eficacia en el personal satisfecho y motivado en su actuar profesional. Las organizaciones, del contexto actual, se basan en la teoría institucional, la cual considera que el conjunto de valores, normas o creencias de la institución son los que guían su comportamiento futuro; en otras palabras, son los factores institucionales los que contribuyen al éxito de la organización (García, Vilalta y Perdono, 2014), por ello, es importante que los administradores de las instituciones identifiquen estos factores para modificarlos o controlarlos (Veblen, 1899; citado por Nelson, 2001).

La relación baja, directa y significativa reportada entre los factores institucionales y la satisfacción laboral corrobora la teoría de Herzberg, quien menciona que la ausencia de estos factores generan insatisfacción, mientas que la presencia de ellos motiva escasamente y también refuerza la teoría institucional, pues al estar presente los factores de higiene no existe insatisfac- 
ción y, por ende, va a contribuir a una mayor eficiencia y eficacia en el logro de los objetivos institucionales, lo cual implica una mejora en los resultados de la organización y, en definitiva, un aporte al incremento en el valor económico (Borra y Gómez, 2012).

Aunque la literatura considera que los factores institucionales tienen relevancia explicativa en el comportamiento de la organización (Jiménez y Narbona, 2010), es importante tener en cuenta que el aumento de la satisfacción laboral del profesional optimiza la relación con el paciente, y con ello se incrementa la calidad de los cuidados prestados (Ruiz, J. E. 2001, citado por Ruzafa 2008), además, se debe contribuir de manera significativa al éxito de la institución, en este caso, del Hospital María Auxiliadora donde se realizó el estudio. Se sugiere tratar estos factores cuidadosamente debido a la independencia en su aparición, comportamiento y las distintas formas cómo afectan la conducta del individuo (Huber 2000), como en los profesionales de enfermería.

Se encontró relación directa y significativa entre los factores institucionales y la satisfacción laboral; asi- mismo, entre los factores institucionales de salario, condiciones laborales, políticas administrativas y la satisfacción laboral de los profesionales de enfermería del Hospital María Auxiliadora. No existe relación directa y significativa entre los factores institucionales de supervisión, relaciones interpersonales y la satisfacción laboral de los profesionales de enfermería del Hospital María Auxiliadora.

\section{Declaración de financiamiento y de conflicto de intereses:}

El estudio fue financiado por los autores, quienes declaran no tener algún tipo de conflicto de interés en la investigación realizada.

\section{Correspondencia:}

Nancy Huamán Salazar

EP Enfermería, Facultad de Medicina. Universidad Nacional Mayor de San Marcos. Av. Grau Nº 755. Lima. Perú.

e-mail: nhuamans@hotmail.com

\section{REFERENCIAS BIBLIOGRÁFICAS}

Arredondo-González, C. P., y Siles-González, J. (2009). Tecnología y Humanización de los Cuidados: Una mirada desde la Teoría de las Relaciones Interpersonales. Index de Enfermería, 18(1), 32-36. Recuperado de http://scielo.isciii.es/scielo.php?script=sci arttext\&pid=S1132-12962009001100007

Balderas, M. (2009). Administración de los servicios de enfermería (5 $5^{\mathrm{a}}$ Ed.). México. Mc Graw Hill.

Bellido, M. C. (2000). Políticas empresariales de conciliación vida familiar y laboral: buenas prácticas. Formación y acreditación en consultoría para la igualdad de mujeres y hombres, 1-31. Recuperado de http://www.unav. edu/matrimonioyfamilia/observatorio/uploads/22093 Caballero_Buenas-practicas-2000.pdf.

Borra, C. y Gómez, F. (2012). Satisfacción laboral y salario: ¿compensa la renta laboral las condiciones no monetarias del trabajo? Revista de economía aplicada. 20(60), 2551. Recuperado de http://www.revecap.com/revista/ numeros/60/borra_gomez.html

Chen, Chung-Jen y Huang, Jing-Wen. (2007). How organizational climate and structure affect knowledge management. The social interaction perspective. International Journal of Information Management. 27(2), 104-118. DOI: 10.1016/j.ijinfomgt.2006.11.001.

Chiavenato. I. (2002). Gestión del talento humano. El nuevo papel de los recursos humanos en las organizaciones. Bogotá, Colombia: McGraw-Hill.
Cifuentes, J. E. (2012). Satisfacción laboral en enfermería en una Institución de salud de cuarto nivel de atención (Tesis de Maestría, Universidad Nacional de Colombia). Recuperada de http://www.bdigital.unal.edu.co/8907/

Cisneros Centeno, C. (2011). Satisfacción laboral del personal de enfermería y su relación con las condiciones de trabajo hospitalario (Doctoral dissertation, Facultad de Enfermería). Recuperado de http://ninive.uaslp.mx/ jspui/bitstream/i/3020/4/MAE1ASL01101.pdf.

Edwards, J. y Cable, D. (2009). The value of value congruence. Journal of Applied Psychology. 94 (3), 654677. DOI: 10.1037/a0014891

Ferres, N., Connell, J. y Travaglione, A. (2004). Co-worker trust as a social catalyst for constructive employee attitudes. Journal of Managerial Psychology. 19 (6), 608-622. DOI: 10.1108/02683940410551516

García Flores, B. K., \& Vilalta y Perdomo, C. J. (2014). Los factores que incrementan la probabilidad de éxito de las iniciativas fiscales federales en México, 19972009. Política y gobierno, 21(2), 253-289. Recuperado de http://www.scielo.org.mx/scielo.php?script=sci_ arttext\&pid=S1665-20372014000200001

Gómez-Cardona, J., Becerra-de Anda M.L. Beltrán-Márquez, B.E., García-L. J.M., Gómez-Olmos, M.T. (2012). Satisfacción laboral del personal de enfermería del área administrativa. Revista de Enfermería del Instituto Mexicano del Seguro Social. 22(3), 5-9. Recuperado de 
http://www.medigraphic.com/pdfs/enfermeriaimss/eim2012/eim121b.pdf

Huber, D. (2000). Liderazgo y administración en enfermería ( $2^{\mathrm{a}}$ Ed.). México: McGraw-Hill Interamericana.

Iona Gherman, T., Iturbe Vásquez, J. A., y Osorio Murillo, D. L. (2013). La teoría motivacional de los dos factores: un caso de estudio (Tesis de Maestría, Pontificia Universidad Catòlica del Perú). Recuperado de http://tesis.pucp.edu. pe/repositorio/handle/123456789/4555

Ivancevich, J. M., Konopaske, R., y Matteson, M. T. (2006). Comportamiento organizacional $\left(7^{\mathrm{a}}\right.$ Ed.). México: McGraw-Hill.

Jiménez, J. C., \& Narbona, A. (2010). Los factores institucionales como determinantes de los flujos comerciales internacionales. Revista de Economía Mundial. 24, 23-48. Recuperado de http://rabida.uhu.es/ dspace/handle/10272/3578

Leguizamón, L. y Gómez, V. (2002). Condiciones laborales y de salud en enfermeras de Santafé de Bogotá. Revista International Journal of Clinical and Health Psychology. 2(1), 173-182. Recuperado de http://www.redalyc.org/ articulo.oa?id=33720109

Luthans, F. (2008). Comportamiento organizacional $\left(11^{\mathrm{a}}\right.$ Ed.). México: McGraw-Hill.

Marriner, Ann (1993). Manual para administración de enfermería (4a Ed.). México Mc.Graw-Hill.

Martínez, H., Rodríguez, C. R., \& García, E. I. (2009). Satisfacción laboral en enfermeras del hospital de oncología Centro Médico Nacional Siglo XXI IMSS. Revista de enfermería universitaria ENEO-UNAM, 6, 21. Recuperado de http://www.medigraphic.com/pdfs/ enfuni/eu-2009/eu094d.pdf

Méndez, C. A. (2009). Los recursos humanos de salud en Chile: el desafío pendiente de la reforma. Revista Panamericana de Salud Pública, 26(3), 276-280. DOI: 10.1590/S1020-49892009000900012

Nelson, R. R., y Sampat, B. N. (2001). Making sense of institutions as a factor shaping economic performance. Revista de Economía Institucional, 44(1), 31-54. DOI: 10.1016/S0167-2681(00)00152-9
Ortega, Y. G. (2007). La enfermera experta y las relaciones interpersonales. Aquichan. 7(2), 130-138. Recuperado de http://www.redalyc.org/articulo.oa?id=74107205

Perazzi, J. R. (2007). Estudio comparativo de salarios entre trabajadores públicos y privados en Venezuela. Caracas: Banco Central de Venezuela.

Ramírez, R., Abreu, J.L. y Badii M.H. (2008). La motivación laboral, factor fundamental para el logro de los objetivos organizacionales: Caso empresa manufacturera de tubería de acero. International Journal of Good Conscience, 3(1), 143-185. Recuperado de http://www. spentamexico.org/v3-n1/3(1)\%20143-185.pdf

Robbins, S.P. Judge, T.A. (2013) Comportamiento organizacional (15 Ed.). México: Editorial Pearson.

Romero, O. (1999). Crecimiento psicológico y motivaciones sociales ( $3^{\mathrm{a}} \mathrm{Ed}$.). Mérida: Rogya.

Ruzafa-Martínez, M., Madrigal-Torres, M., Velandrino -Nicolás, A., y López-Iborra, L. (2008). Satisfacción laboral de los profesionales de enfermería españoles que trabajan en hospitales ingleses. Gaceta Sanitaria. 22(5), 434-442. Recuperado de http:// scielo.isciii.es/scielo.php?script=sci_arttext\&pid =S0213-91112008005500007

Spector, P (1997). Job Satisfaction. Application, assessment, cause, and consequences. California: Sage Publications.

Tan, H. y Lim, A. (2009). Trust in coworkers and trust in organizations. The Journal of Psychology. 143 (1), 4566. DOI: 10.3200/JRLP.143.1.45-66

Umaymas, Y. (2003). Personal Characteristics and job satisfaction among nurses in Lebanon. Journal Nursing Advanced. 33(7/8), 384-390. Recuperado de http://journals. lww.com/jonajournal/Abstract/2003/07000/Personal_ Characteristics_and_Job_Satisfaction.6.aspx?trendmdshared $=0$

Vinagre, J., Heredero, C. (2013). Impacto estratégico de las guías de práctica clínica en enfermería en la función de gestión de la supervisión. Rev Esc Enferm USP, 47(5):1241-6. 